\title{
Walking Stroop carpet: an innovative dual-task concept for detecting cognitive impairment
}

This article was published in the following Dove Press journal:

Clinical Interventions in Aging

19 March 2013

Number of times this article has been viewed

\section{A Perrochon ${ }^{1,2,5}$ \\ G Kemoun ${ }^{1,2}$ \\ E Watelain ${ }^{3,4}$ \\ A Berthoz ${ }^{5}$}

'ISIS, Research Institute on Handicap and Aging, Paris; ${ }^{2}$ Université de Poitiers, Laboratoire Mobilité, Vieillissement et Exercice (MOVE), EA 63|4, ${ }^{3}$ Université de Valenciennes, LAMIH, UMR CNRS 820I, ${ }^{4}$ Université Sud Toulon Var, HandiBio, EA 4322, La Garde, ${ }^{5}$ LPPA, UMR CNRS 7I52, Collège de France, Paris, France
Correspondence:Anaick Perrochon MOVE, EA 63।4, University of Poitiers, 8 allée Jean Monnet, 86000 Poitiers, France $\mathrm{Tel}+3306$ | | 4608 |3 Fax +330156580078 Email anaick.perrochon@hotmail.fr gilles.kemoun@wanadoo.fr
Background: Several studies have reported the potential value of the dual-task concept during locomotion in clinical evaluation because cognitive decline is strongly associated with gait abnormalities. However, current dual-task tests appear to be insufficient for early diagnosis of cognitive impairment.

Methods: Forty-nine subjects (young, old, with or without mild cognitive impairment) underwent cognitive evaluation (Mini-Mental State Examination, Frontal Assessment Battery, five-word test, Stroop, clock-drawing) and single-task locomotor evaluation on an electronic walkway. They were then dual-task-tested on the Walking Stroop carpet, which is an adaptation of the Stroop color-word task for locomotion. A cluster analysis, followed by an analysis of variance, was performed to assess gait parameters.

Results: Cluster analysis of gait parameters on the Walking Stroop carpet revealed an interaction between cognitive and functional abilities because it made it possible to distinguish dysexecutive cognitive fragility or decline with a sensitivity of $89 \%$ and a specificity of $94 \%$. Locomotor abilities differed according to the group and dual-task conditions. Healthy subjects performed less well on dual-tasking under reading conditions than when they were asked to distinguish colors, whereas dysexecutive subjects had worse motor performances when they were required to dual task.

Conclusion: The Walking Stroop carpet is a dual-task test that enables early detection of cognitive fragility that has not been revealed by traditional neuropsychological tests or singletask walking analysis.

Keywords: stroop, dual-task, walking, mild cognitive impairment

\section{Introduction}

Several authors have reported a causal relationship between the single-task motor abilities of older subjects and an advanced stage of dementia. Analysis of single-task walking as an evaluation tool and prediction of dementia pathology represents a recent approach in the literature. ${ }^{1,2}$ However, clinical and therapeutic interest appears to be more in early detection of mild cognitive impairment, given that this is often associated with the prodromal phases of several dementia pathologies, eg, Alzheimer's disease, and frontotemporal and vascular dementia. The annual rate of conversion from mild cognitive impairment to dementia ranges from $5 \%$ to $50 \%$, depending on the cognitive profile of subjects and the etiology of dementia. ${ }^{3}$ Several authors have reached a consensus, placing the concept of mild cognitive impairment within a stable nosologic framework (mnesic complaint by the patient or his/her family members without repercussions on daily life, an absence of dementia), despite the heterogeneity 
of its population. ${ }^{4,5}$ In 2011, Erk et $\mathrm{al}^{6}$ demonstrated that mnesic complaints in older patients were associated with structural and functional modification of the hippocampus, whereas cognitive impairment has yet to be objectified by neuropsychological tests. Therefore, diagnosis of mild cognitive impairment more particularly relies on early detection of dysexecutive and mnesic impairment. However, traditional tests appear to be inadequate for early detection of cognitive impairment at high-risk of progression to dementia. There are multiple reasons for this, ie, use of neuropsychological tests that are not sensitive enough, unsuitable norms, cognitive reserve, and/or underestimated compensation mechanisms.

The relationship between motor performance and cognitive ability can be investigated using the dual-task concept. Identification of cognitive resources (executive function or attention) engaged during dual-tasking triggers a reduction in walking speed in older subjects, ${ }^{7-10}$ and even cessation of walking in subjects with Alzheimer's disease. ${ }^{11}$ Executive function refers to a variety of higher cognitive processes. Several authors have demonstrated the value of analysis of walking stability and variability as a tool for detection of cognitive impairment, beginning at the mild cognitive impairment stage because the motor profiles of these subjects have similarities to those of patients with Alzheimer's disease in single-tasking ${ }^{12}$ and dual-tasking, ${ }^{13}$ whereas their motor abilities are significantly different from those in young and older healthy subjects for single-tasking and dual-tasking. ${ }^{14-16}$

In everyday activities, there are numerous dual-task locomotor situations that require active involvement of the visual system. Nevertheless, few studies have been performed on the ability to walk according to visuospatial information. In 2011, Al Yahya et al ${ }^{17}$ described numerous dual-tasking situations based on working memory exercises, decisionmaking, and verbal fluency. Likewise, they enumerated various spatiotemporal parameters of walking used to evaluate dual-task motor performance (eg, speed, frequency, length of stride). Executive function is often implicated in dualtask situations because subjects must walk and adapt to new and/or complex situations. These include several cognitive situations, such as working memory, mental inhibition, and mental flexibility. ${ }^{18}$

In 2008, Yogev-Seligmann et a ${ }^{19}$ proposed a theoretical framework showing how changes in different aspects of executive function (volition, self-awareness, planning, inhibition of dominant response and external distraction during response control, dual-task coordination) can modify gait patterns. They also reviewed studies indicating a significant link between gait and executive function, in particular, during dual-task situations. However, most studies of dual-tasking have been limited to evaluation of linear gait and are associated with one of two verbal tasks, ie, counting backwards (working memory) or listing animal names (semantic memory). ${ }^{13,15,16,20,21}$ Therefore, other cognitive functions, such as the mental inhibition concept in dual-tasking, have been little studied. Nevertheless, in 2007, Fournet et al ${ }^{22}$ reported early deficits in inhibition at an attentional level in subjects with mild cognitive impairment. The increased sensitivity to interference seen in such individuals is commonly revealed during the Stroop test. This selective attention task, which consists of inhibiting an automatic verbal response, is markedly reduced in older people with mild cognitive impairment compared with healthy older people. ${ }^{23-25}$ On the other hand, while there are numerous adaptations of the Stroop test based on the interference effect proposed by Stroop in 1935, very few studies have been done using the Stroop test in the context of dual-tasking, ${ }^{26-28}$ and to our knowledge, none has used a spatial navigation task. However, several authors have shown that an environmental version of Stroop provides a better indication of the cognitive abilities of subjects compared with standard tests. ${ }^{29,30}$

The aim of this work was to devise a dual-task test that can be used for early detection of mild cognitive impairment. By adapting the Stroop color word test to walking, we also attempted to determine if the ability to integrate visual information during dual-tasking could be modified by the effects of aging and/or cognitive status.

We hypothesized that a new dual-task approach could refine the diagnosis of cognitive impairment in its very early stages, which cannot be detected by a single-task walking test or by a traditional paper-pencil neuropsychological test. It would then follow that older subjects with mild cognitive impairment have an altered ability to integrate visual information during dual-tasking, indicated by a reduction in their dual-task walking performance.

\section{Materials and methods Population}

Our study population was made up of 14 young subjects (mean age 21.1 [20-30] years), 18 older adults (mean age 69.3 [64-74] years), and 17 very old adults (mean age 81.3 [75 onwards] years). The subjects over 65 years of age were recruited from cultural associations. Inclusion criteria were: willingness to participate in the study, living in a noninstitutional environment, and ability to get around without technical assistance on a daily basis. Exclusion criteria were: a cognitive complaint with repercussions on 
daily functional ability, uncorrected visual impairment, neurologic pathology (eg, Parkinson's disease, stroke), orthopedic surgery to the lower limbs, depression, and being on medication that could influence posture and/or gait.

\section{Clinical and cognitive evaluation}

Subjects were assessed by a doctor for inclusion and exclusion criteria. All of the subjects then underwent a battery of rapid cognitive impairment tests by a neuropsychologist using the Mini Mental State Examination ${ }^{31}$ and the Frontal Assessment Battery. ${ }^{32}$ Mood and depression were tested using the Geriatric Depression Scale. ${ }^{33}$ Daily and functional activities were also evaluated using the Instrumental Activities of Daily Living test. ${ }^{34}$

Executive and mnesic functioning was evaluated by the Wechsler Adult Intelligence Scale (WAIS) III, ${ }^{35}$ the Trail Making Test (TMT), ${ }^{36}$ the Stroop color-word test, ${ }^{37}$ and the five-word test. ${ }^{38}$ Only the working memory part of the WAIS III was retained for its ability to formulate and resolve mental problems. The TMT is a pencil-paper test which consists of linking targets as quickly as possible in numeric order (1-25) in Part A and alternating between numbers and letters in Part B. It evaluates visual attention in Part A and mental flexibility in Part B. The Stroop color-word test is a test of mental inhibition of selective attention. It is made up of three parts. The first part consists of reading the names of colors printed in black ink; in the second part, the subject is asked to name colors in rectangles; and in the third part, the subject reads the color of the words aloud. The subject must name a maximum of items for each page in 45 seconds. The five-word test is a rapid mnesic test using five words with immediate and deferred recall which can be indexed.

\section{Single-task walking}

We performed a motor evaluation of single-task spontaneous walking for $10 \mathrm{~m}$ in a normal environment on an $8 \mathrm{~m}$ electronic walkway (GAITRite ${ }^{\circledR}$, CIR Systems Inc, Sparta, NJ, USA). This tool is equipped with a portable, pressure-sensitive electronic walkway $(793 \mathrm{~cm} \times 61 \mathrm{~cm} \times 0.6 \mathrm{~cm}[\mathrm{~L} \times \mathrm{W} \times \mathrm{H}])$, so recording of spatiotemporal gait parameters does not include the acceleration and deceleration phases. In order to have a value that is representative of single-task motor ability, subjects were asked to walk five times at their own pace.

\section{Dual-task walking}

We used an adapted version of the Stroop color-word paperwalking test (dual-task) with the Walking Stroop carpet (WSC) in order to create a visuospatial dual-task based on the Stroop interference effect (Figure 1A). This test was developed by our team in our motion analysis laboratory. The WSC is a dual-task motor test during which the subject steps on targets on a $5 \mathrm{~m}$ long removable cloth support on an electronic walkway. This system records all gait parameters during Stroop visuospatial adaptation to dual-tasking. Each removable support is associated with different pages of the Stroop color-word test. However, the methodological constraints of this dual-task test have brought about modifications to the original version. The principal adaptation is the form of restoration of the dual-task response. In fact, the WSC requires a motor response because the subject must step on targets on the ground whereas the response is verbal in the table version. The targets are set up like the Stroop Victoria test, ${ }^{39}$ ie, the stimuli are presented in lines of four different words and/or colors (red, yellow, blue, and green). Before giving the green light, the examiner informs the subject that a word or color will remain unchanged during the entire test. Each line has only one correct answer and thereby forms a circuit of 15 targets over a distance of $5 \mathrm{~m}$. The mean distance between targets of $45 \mathrm{~cm}$ enables all subjects to go from one target to another without difficulty. Each condition is defined so that subject steps do not get crossed (Figure 1B-D).

The conditions are associated with the stages of the Stroop color-word test and performance of the test is always in the same order. Thus, there is one congruent situation followed by two incongruent situations. The first condition, $\mathrm{DT}_{\mathrm{B} \& \mathrm{~W}}$ (dualtask $_{\text {black\&white }}$ ) consists of walking on the word corresponding to the name of a color (Figure 1B). If the subject is supposed to walk on the word "green", on each line, he/she must spot the target and refrain from reading the other words (red, yellow, blue). In this condition, the carpet is black and the targets are

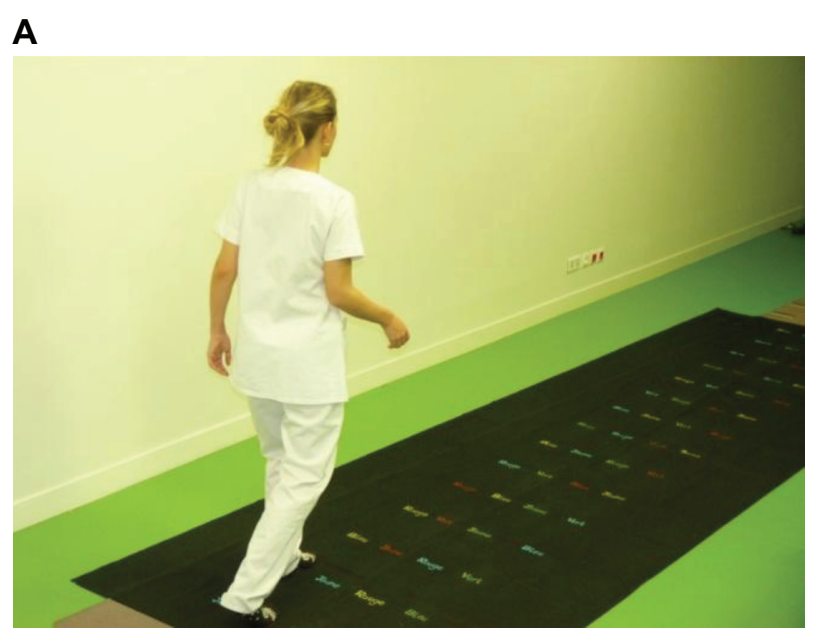

Figure IA Illustration of the experimental condition in the "Walking Stroop carpet". 


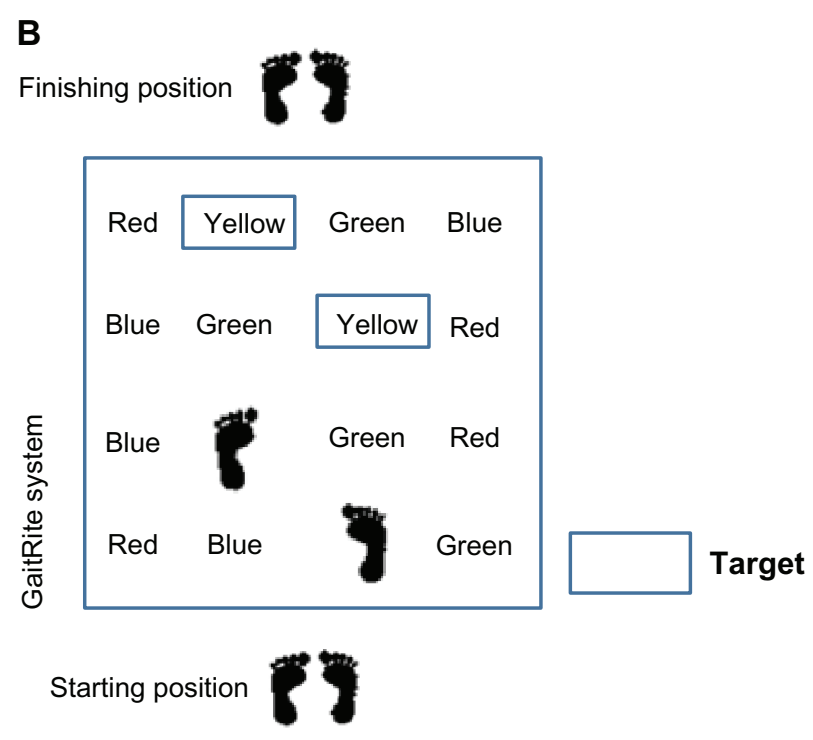

Figure IB First condition: $\mathrm{DT}_{\mathrm{B} W}$.

Note: In that condition, the subject has to read and to walk on the word "yellow".

white in order to increase the contrast and reduce the risk of instability linked to the treatment of information. Indeed, in 1991, Lord et $\mathrm{al}^{40}$ demonstrated that a reduction in the ability to distinguish characters with low contrast was a strong predictor of the risk of falling. The second condition, $\mathrm{DT}_{\text {word }}$ consists of walking on a word that is written in several different colors (Figure 1C). If the instructions are to walk on the word "blue", the subject must avoid the color of the word and the other words (green, yellow, red). The third condition, DT color $_{\text {is }}$ the paper-pencil test interference situation, which consists of walking on words written in a color and avoiding reading the

\section{C} Finishing position

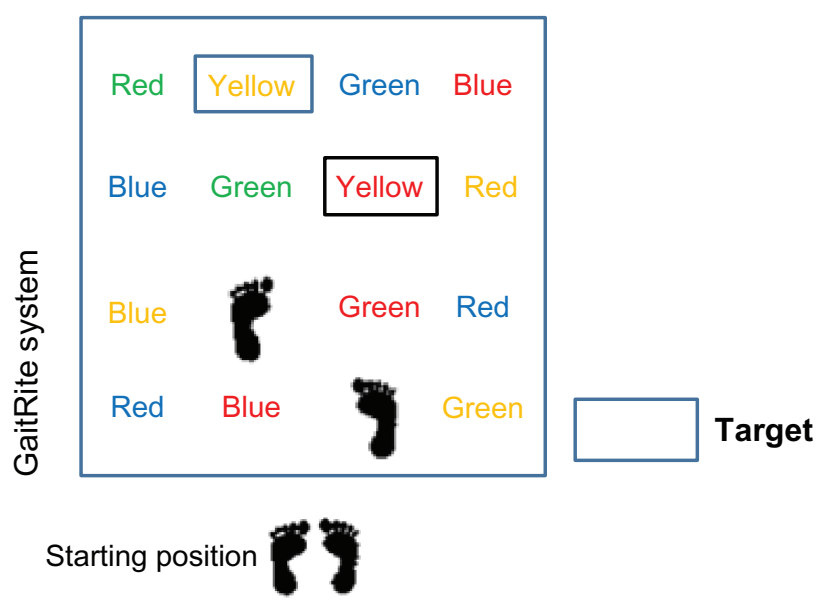

Figure IC Second condition: DT ${ }_{\text {Word }}$.

Note: In that condition, the subject has to read and to walk on the word "yellow".
Finishing position

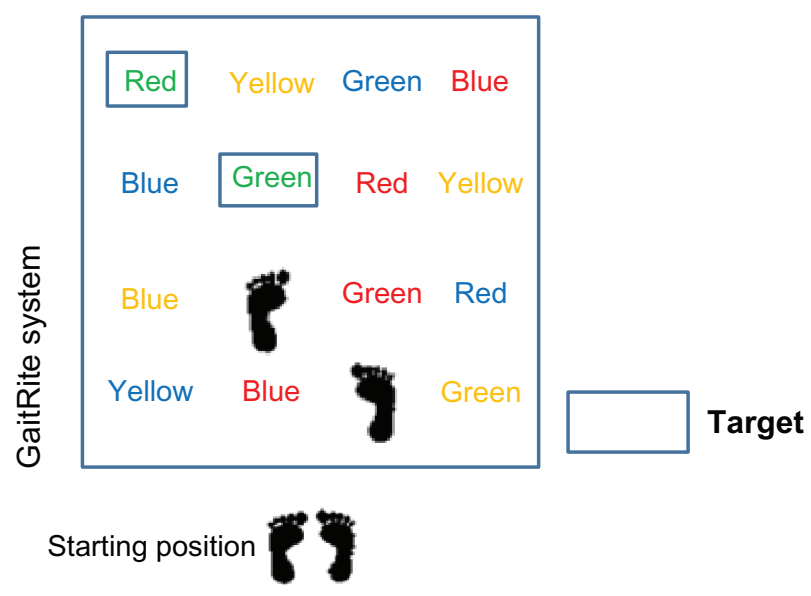

Figure ID Third condition: DT

Note: In that condition, the subject has to read and to walk on the color "green".

words (Figure 1D). If the instructions are to walk on the color red, the subject must avoid reading the words and not walk on the other colors (blue, yellow, green). In this condition, the targets on the colored carpet can be congruent (the word "red" is written with red ink) or incongruent (the word "green" is written in red ink) in the second and third conditions. We asked subjects to perform the test under each condition twice, ie, reading $\mathrm{DT}_{\mathrm{B} \& \mathrm{~W}}$ reading colored $\mathrm{DT}_{\text {word }}$ and spotting $\mathrm{DT}_{\text {color }}$

Subjects received the following instruction to perform the two tasks as best they could: "Walk as fast as possible and do the Stroop exercise without making any mistakes." However, no priority was given to one domain or the other. Each condition had a familiarization and learning phase over a distance of $2 \mathrm{~m}$, which was repeated until the subject understood the task to be accomplished (a maximum of three repetitions were necessary). During the test, the result was correct from the moment the subject placed a part of his/her foot on the correct target. Each condition was filmed by a webcam $(640 \times 480)$ that was synchronized with the electronic walkway in order to detect errors accurately. Because the positions of the targets were predetermined according to the task, the subjects were not able to retain their usual gait parameters such as stride length, distance, and variability of stride. Therefore, we were able to focus on the following parameters: velocity, frequency, time in double support, gait cycle time, and number of steps.

\section{Statistical analysis}

In the first instance, the aim was to detect the possible existence of types of behavior linked to the motor task. This required a statistical analysis in order to assess the groups 
solely on their gait characteristics during single-tasking and/or dual-tasking. The ascendant hierarchic classification method or cluster analysis (Ward's method, ${ }^{41}$ Euclidean distance) regroups subjects according to their functional similarities by using spatiotemporal parameters of walking during single-tasking and dual-tasking. We averaged five tests in single-tasking and two tests for each dual-tasking condition to obtain a single representative value per variable and per walking condition in the cluster analysis. A one-way analysis of variance followed by a post hoc Tukey's test was then used to determine the differences between the groups for the different gait parameters. Next, a Student's $t$-test of the different neuropsychological tests was performed to reveal any differences in the cognitive profiles for each group.

The differences between dual-tasking conditions and gait parameters for each group were determined by one-way repeated-measures analysis of variance followed by a post hoc Tukey's test. The statistical analysis was performed using Statistica version 9 (Statsoft Inc, Tulsa, OK, USA) software with a significant difference if $P<0.05$.

The sensitivity and specificity of the WSC to detect subjects with mild cognitive impairment were calculated as follows:

Sensitivity $=$ Number of mild cognitive impairment subjects with low performance WSC (cf infra)/Total number of mild cognitive impairment subjects

Specificity $=$ Number of healthy subjects with high performance WSC (cf infra)/Total number of healthy subjects.

Low performance refers to the motor performance of subjects in the group with the largest deficit in WSC, and high performance refers to the group with the best gait parameters in dual-tasking. Given that this test is new and no similar study has been reported, there are no reference values.

\section{Results}

\section{Cognitive assessment}

The results of the neuropsychological Instrumental Activities of Daily Living and Geriatric Depression Scale tests identified a group of 25 healthy subjects (14 young, 11 older or elderly healthy adults) and 15 subjects with mild cognitive impairment who were divided into subgroups according to the criteria devised by Petersen, ${ }^{4}$ ie, six with amnesic mild cognitive impairment (aMCI), three with nonamnesic mild cognitive impairment and impairment of executive function only (naMCI), and six with multiple-domain amnesic mild cognitive impairment (mdMCI). We noted and differentiated nine subjects without cognitive decline but whose performance on executive function tests was always at the limit of the pathologic threshold. These subjects formed another group deemed to have borderline mild cognitive impairment (blMCI), given their common characteristics. ${ }^{30}$

\section{Single-task walking}

The first cluster analysis (Figure 2) was performed on the five single-task parameters (velocity, frequency, number of steps, gait cycle time, time in double support) which were identical to those retained for dual-task conditions. Before proposing a classification on the basis of dual-task conditions, it was important to analyze these same parameters during single-task conditions in order to determine their impact on the formation of groups. For this cluster analysis, we did not differentiate distinct branches, the $\mathrm{R}$ ratio calculation, ${ }^{42} \mathrm{did}$ not identify groups, and the Euclidean distance was too small to match subjects by their motor performances in homogeneous groups. Therefore, these gait variability and stability parameters failed to identify distinct groups.

\section{Dual-task walking}

The second cluster analysis (Figure 3) sought a relationship between single-task and dual-task WSC parameters. Classification using the ascendant hierarchic method revealed two distinct branches, with a Euclidean distance of less than 20 and a reunification at 55 (Figure 3). The $\mathrm{R}$ ratio calculation ${ }^{40}$ identified two distinct walking patterns from all of the subjects' results (limited to 10).

The two groups were functionally differentiated by the overall effect of analysis of variance $(\mathrm{F}=9.2 ; P<0.001)$. Group 1 subjects (with diminished executive function or cognitive fragility) walked more slowly than group 2 subjects (healthy subjects with or without mild amnesic cognitive impairment) under single-task conditions $(\mathrm{F}=4.97$; $P=0.03)$ and under dual-task conditions $(\mathrm{F}=56.71$ to 64.14; $P<0.001$, Figure 4A). There were no differences between the groups for the other single-task parameters. In dual-tasking, motor performances in group 1 were lower than those in group 2 (Figure 4A, B, D, and E), except for the number-of-steps parameter, where there was no significant difference (Figure 4C). However, the number of steps could indirectly provide an indication of the number of errors, but we noted that group 1 subjects did not make more errors than subjects in group $2(\mathrm{~F}=3.21, P=0.12$, Table 1). 


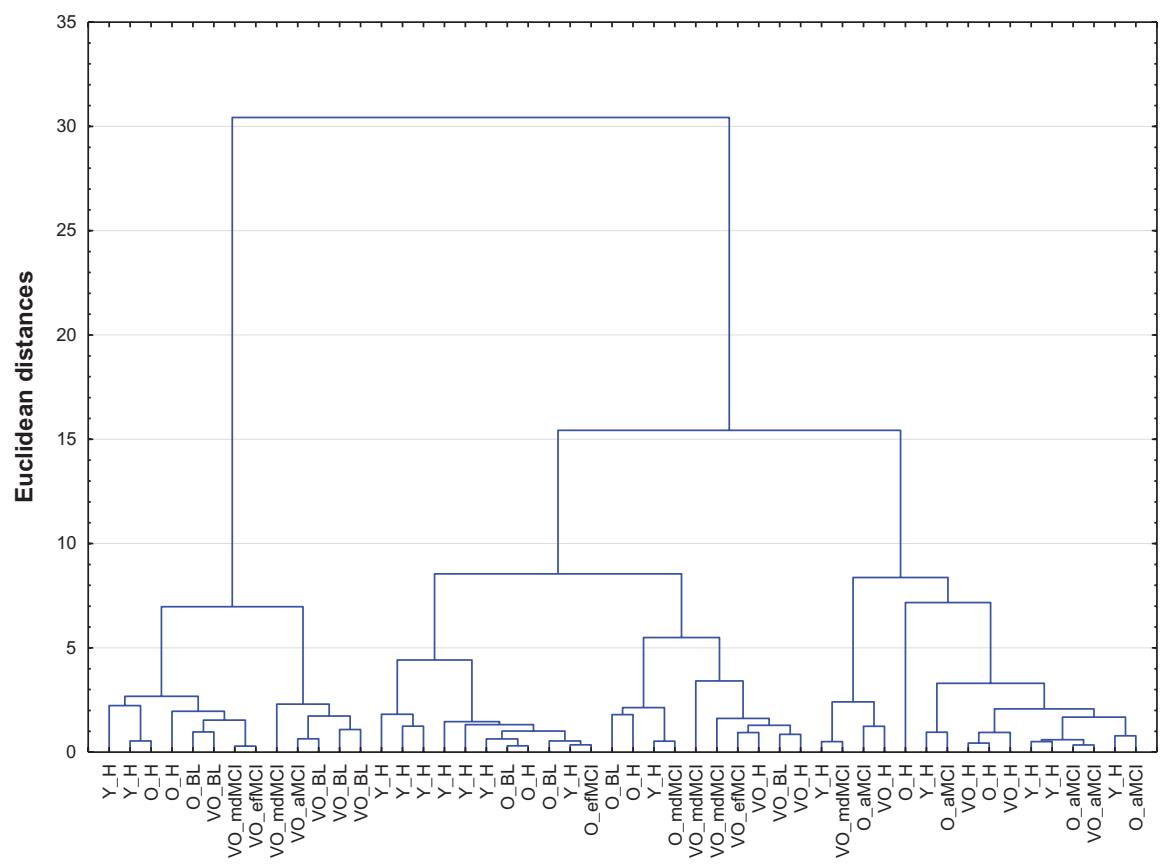

Figure 2 Gait pattern Dendogram of the ST.

Notes: The cluster analysis consists in pairing the individuals based on the similarities of their overall gait parameters and in progressively grouping subjects until we have one group that gathers all the participants.

Abbreviations: Y, Young; O, Older; VO, Very Older; H, Healthy; $\mathrm{BL}$, Borderline $\mathrm{MCl}$; naMCl, non-amnesic $\mathrm{MCl}$ executive impairment only; aMCl, amnesic $\mathrm{MCl}$; $\mathrm{mdMCl}$, multiple-domain amnestic $\mathrm{MCl}$.

\section{Detection of mild cognitive impairment}

The second cluster analysis revealed the existence of two groups. We studied the cognitive performance of each individual in order to determine if the groups formed principally from gait parameters were also differentiated on a cognitive level (Table 2). Group 1 included 18 subjects (seven older adults, 11 very old adults) with diminished executive function or cognitive fragility (one healthy older subject, six with mdMCI, seven with blMCI, three with naMCI, and one with aMCI). Group 2 included 31 subjects (14 young adults, 11 older adults, six very old adults) with or without mild amnesic cognitive impairment ( 24 healthy subjects, two with blMCI, and five with aMCI). The subjects were grouped by motor performance, which appeared to be directly linked to their cognitive ability. However, the results showed that this task does not depend on the effects of aging (old or even very old subjects were mixed with the young subjects in group 2). Subjects in group 1 did not perform well on the WSC because their gait parameters were poor, whereas subjects in group 2 showed better walking performance on the WSC because their gait parameters were significantly better. This dual-task test was able to detect overall cognitive fragility possibly linked to normal cognitive aging or to a predementia state with a sensitivity of $71 \%$ and a specificity of $96 \%$, and detected a change in executive function with a sensitivity of $89 \%$ and a specificity of $94 \%$.

\section{Effect of Stroop during locomotion}

We observed a significant interaction between gait parameters, conditions, and groups $(\mathrm{F}=5.27 ; P<0.001)$. Post hoc analysis did not reveal a significant difference between dual-task conditions for either group with regard to parameters for the number of steps, gait cycle time, or time in double support (Figure 4C-E). A significant difference was found between dual-task conditions for velocity and frequency only in group 2 .

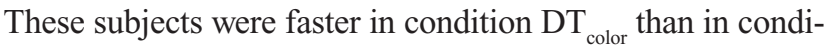
tion $\mathrm{DT}_{\mathrm{B \& W}}(\mathrm{F}=5.27 ; P<0.001)$ and in condition $\mathrm{DT}_{\text {word }}$ $(\mathrm{F}=5.27 ; P<0.001$, Figure 4A). Frequency was also higher in condition $\mathrm{DT}_{\text {color }}$ than in $\mathrm{DT}_{\mathrm{B} \& \mathrm{~W}}(\mathrm{~F}=5.27 ; P<0.001)$ and in $\mathrm{DT}_{\text {word }}(\mathrm{F}=5.27 ; P<0.001$, Figure $4 \mathrm{~B})$. There was no difference between condition $\mathrm{DT}_{\text {color }}$ and the two other conditions for these two parameters in group 1 (Figure 4A and B).

\section{Discussion}

To our knowledge, this is the first study dealing specifically with spatiotemporal gait parameters during visuospatial dual-tasking used to detect mild cognitive impairment. The WSC provides sensitivity (89\%) and specificity (94\%) that is of significant interest in the early detection of predementia dysexecutive impairment.

The WSC test is a complex dual-tasking exercise because the subject must adapt his/her gait and navigate on targets 


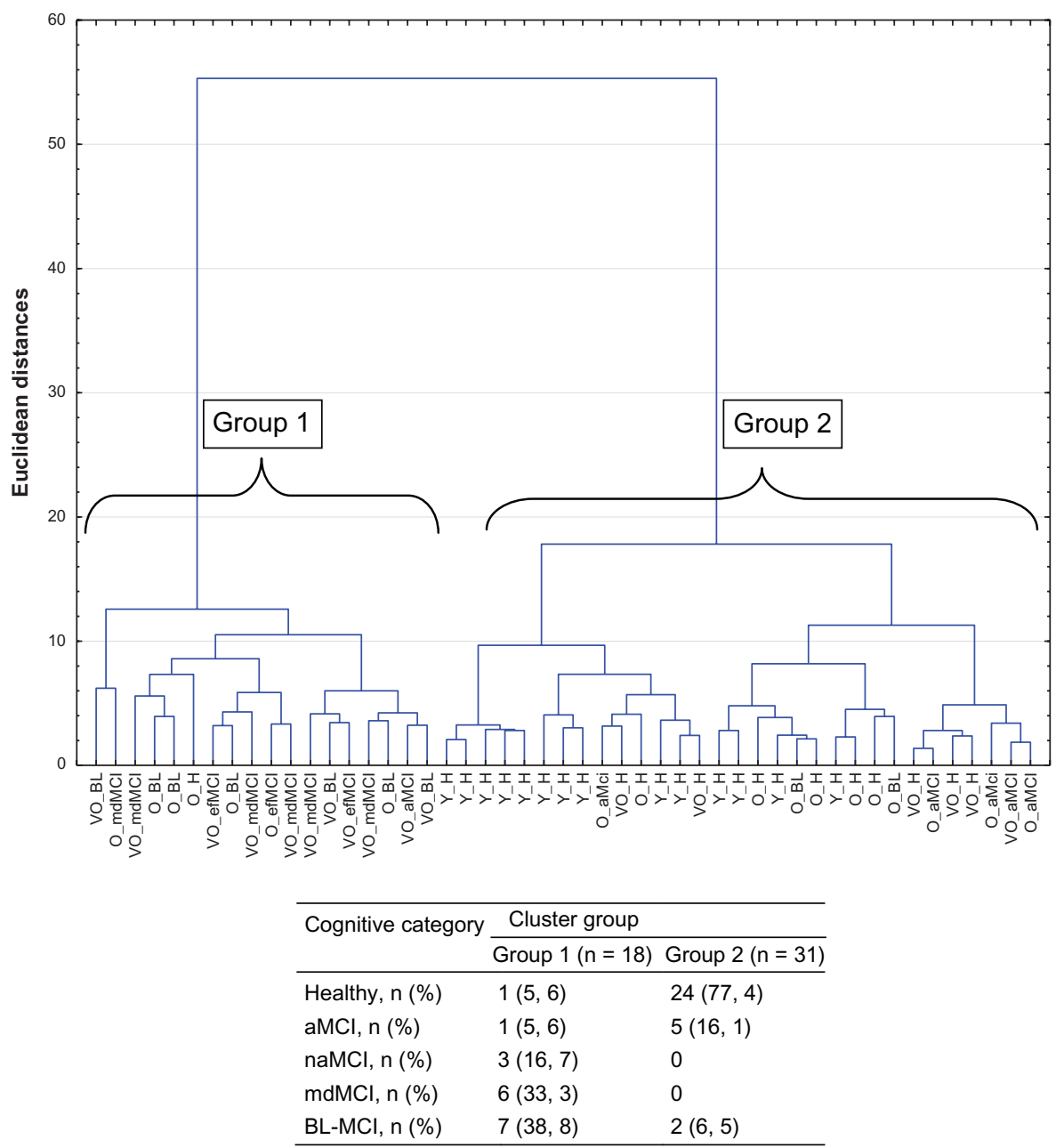

Figure 3 Gait pattern Dendogram of the ST and WSC.

Notes: The figure is read from the bottom where the individual trials are listed, in two families, to top where all gait trials are grouped into a single family. The cluster analysis consists in pairing individuals based on the similarities of their overall gait parameters in dual-task condition (WSC), and in progressively grouping subjects until we have one group that gathers all the participants. The classification through this ascendant hierarchical method highlights a tree structure with two distinct branches brought together within a value of Euclidian distance smaller than 20 for a final merging higher than 50 .

Abbreviations: Y, Young; O, Older; VO, Very Older; $\mathrm{H}$, Healthy; $\mathrm{BL}$, Borderline $\mathrm{MCl}$; naMCl, non-amnesic $\mathrm{MCl}$ executive impairment only; aMCl, amnesic $\mathrm{MCl}$; mdMCl, multiple-domain amnestic $\mathrm{MCl}$.

while mentally inhibiting cognitive information. This cognitive function is known for being particularly sensitive to pathological aging (mild cognitive impairment or Alzheimer's disease). ${ }^{23-25}$ In the present study, as in other studies, adaptation of the Stroop color-word test during environmental situations appeared to provide supplementary information on the cognitive ability of subjects with mild cognitive impairment during dual-tasking ${ }^{30}$ and of healthy subjects during a Stroop task in virtual reality, ${ }^{29}$ when compared with traditional paper-pencil neuropsychological tests. Most studies have approached this issue by seeking to demonstrate significant differences between healthy subjects and those with mild cognitive impairment on a locomotor level during dual-tasking in predetermined groups. ${ }^{13,16,43}$ The drawback for these types of analyses based on group comparisons is that although they reveal a link between executive function and motor ability, they do not come within the logic of detection or early detection of cognitive impairment. Conversely, with the WSC, the second cluster analysis made it possible to detect seven of nine subjects who had early-stage executive function fragility which was not detected by analysis of single-task walking or traditional paper-pencil neuropsychological tests.

Prospective studies over periods of 5 and 6 years in cohorts of 427 and 603 older subjects over 70 years of age demonstrated that initial quantitative measures of gait (velocity, variability, frequency) can predict the risk of developing cognitive impairment or dementia. ${ }^{1,2}$ Verghese et $\mathrm{al}^{2}$ showed that, depending on modified gait parameters, one can even characterize the profile of cognitive impairment 
A

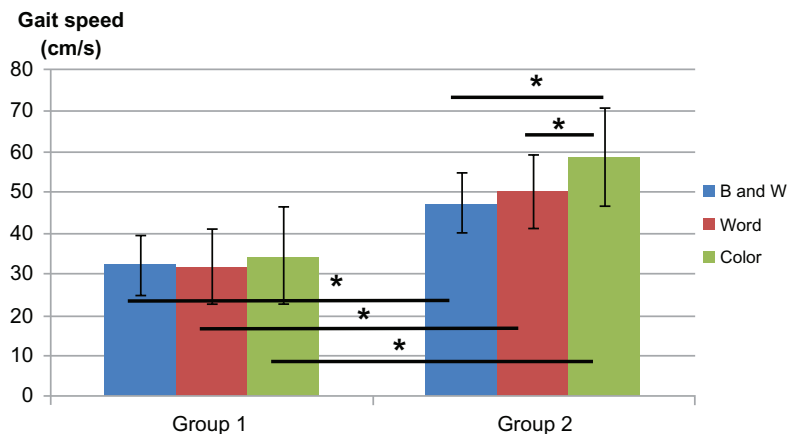

C

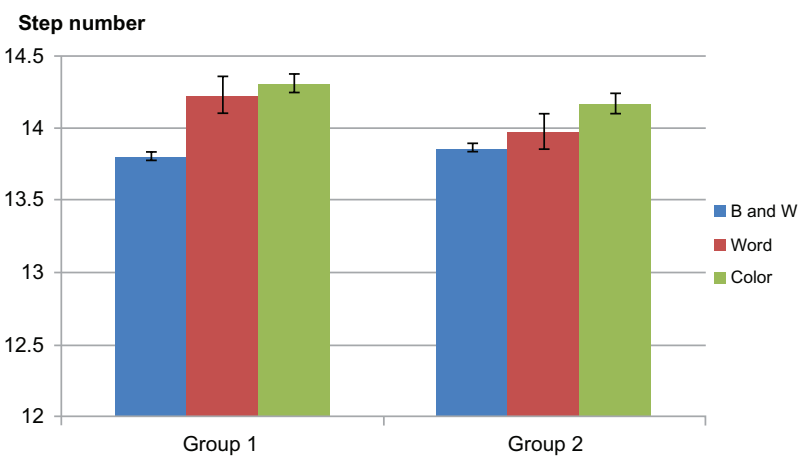

B

Cadence

(step/min)

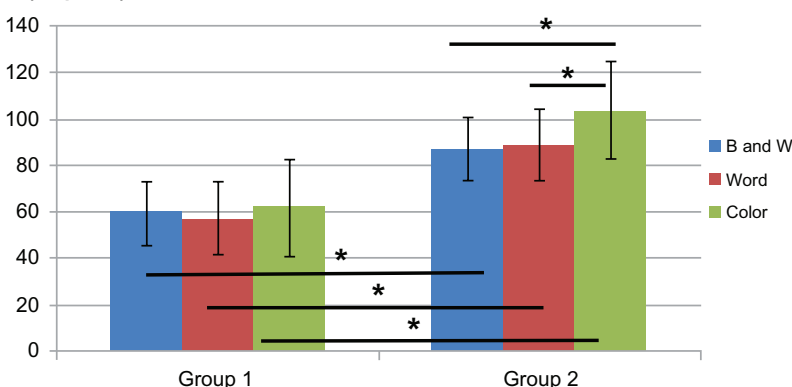

D

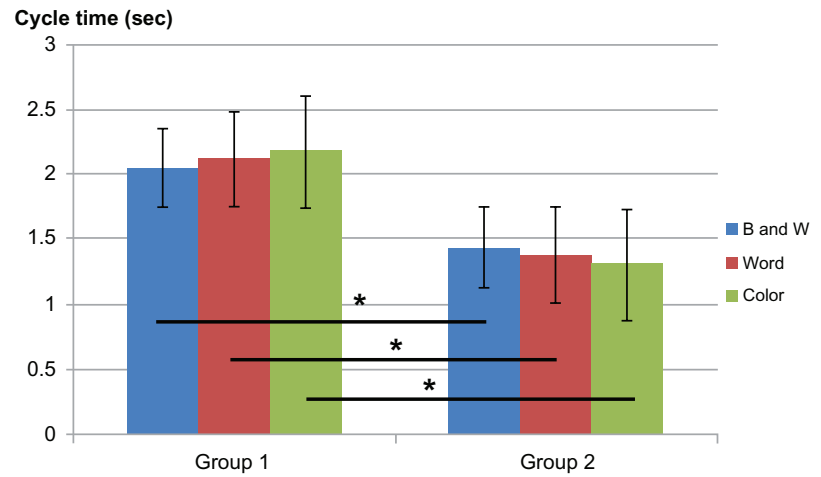

E

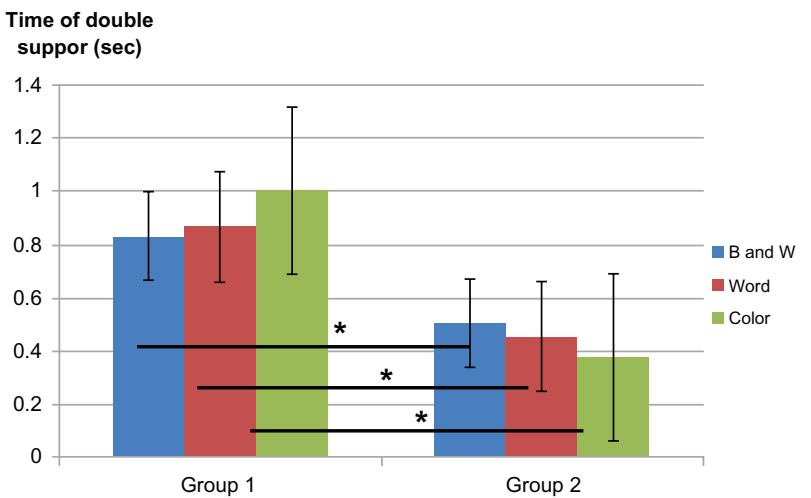

Figure 4 Results of analysis of variance (ANOVA) on gait parameters in each cluster group and walking test condition.

Note: *Significant difference for $P$-value $<0.05$.

(modification of velocity and length of step are factors associated with decline in executive function, whereas the frequency factor determines mnesic deterioration). It is observed that the subjects did not differ in gait assessment in a linear manner, whereas the WSC seems to be a discriminant dual-task to separate out in the elderly those who have dysexecutive problems. On analyzing single-task gait parameters, we did not find factors predictive of cognitive decline (Figure 2). It was only by using the WSC that we found two distinct motor profiles (Figure 3). In addition to distinguishing themselves at a motor level, our two groups of subjects had different levels of cognitive ability. Group 1 subjects have mainly dysexecutive fragility or cognitive impairment diagnosed by paper-pencil neuropsychological tests, whereas group 2 was made up of younger and older subjects of whom only a few had impairment of mnesic function. Depending on their typology, cognitive functions were associated with subject dual-task motor abilities.

This new approach to adaptation of dual-tasking to a neuropsychological test increased the specificity for detection of dysexecutive impairment. Several authors have shown that a deficit in executive function is associated with disruption of walking as part of dual-tasking, ${ }^{14,19}$ and that increasing the complexity of motor tasks enables characterization of the cognitive 
Table I Number of errors in all conditions in DT

\begin{tabular}{llll}
\hline Walking test & \multicolumn{2}{l}{ Cluster group } & P-value \\
\cline { 2 - 3 } condition & Group I & Group 2 & \\
\hline $\mathrm{DT}_{\text {B\&W }}$ & $0.09 \pm 0.3$ & $0.09 \pm 0.39$ & 0.3 \\
$\mathrm{DT}_{\text {word }}$ & $0.08 \pm 0.32$ & $0.03 \pm 0.1$ & 0.24 \\
$\mathrm{DT}_{\text {color }}$ & $0.44 \pm 0.85$ & $0.19 \pm 0.47$ & 0.19 \\
Total & $0.2 \pm 0.29$ & $0.1 \pm 0.28$ & 0.12 \\
\hline
\end{tabular}

Notes: Mean \pm standard deviation, Student's $t$-test. The values shown correspond to the average number of test errors for subjects in each group.

Abbreviation: DT, dual-task.

profile of subjects. ${ }^{14}$ In fact, not all subjects with mild cognitive impairment respond in the same manner to visuospatial dualtask exercises, because the motor performance of subjects with aMCI did not differ from those of healthy subjects during the WSC test or the TMT test by Persad et al. ${ }^{14}$ Mnesic function did not play a significant role in motor performance in certain walking situations. ${ }^{14,44}$ The complexity of the WSC is based on spatial navigation abilities, visual information processing, and cognitive load (mental inhibition). In addition, this exercise causes hesitation in the elderly, which results in increased double support time and corresponds to information processing time and cognitive task performance.

The role of vision in gait control during locomotion has been demonstrated by numerous authors, particularly when the environment is enriched with visual information. ${ }^{45-47}$
Vision is principally used in the support phase when one must plan and execute an optimal step. ${ }^{45}$ During WSC, this mechanism triggers a modification in gait parameters, in particular by a reduction in velocity and frequency, and more so by an increase in double support time. At the same time, Di Fabio et al $^{48}$ suggested that individuals with a reduced cognitive ability have more difficulty identifying the environment and it is necessary for them to fixate more to have a maximum of visual information. The distinction between the motor abilities of healthy (younger or older) and subjects with dementia pathology can also be explained by this strategy to favor the treatment of information during the double support phase because the attentional cost needed to control posture is low and attentional resources are more easily available to perform the cognitive task. The WSC is a complex dual-tasking exercise, the value of which is very early detection of impairment or even cognitive fragility (blMCI) by analyzing motor performance.

The notion of fragility is a recent concept that makes it possible to target a category of older subjects who are physically vulnerable. The cognitive dimension is rarely taken into account. However, these two aspects appear to be intertwined. Hommet and Mondon ${ }^{49}$ stressed the existence of a relationship between executive function and motor fragility. However, few studies have made the link between motor

Table 2 Demographic and clinical characteristics of study participants stratified by cluster results

\begin{tabular}{|c|c|c|c|}
\hline \multirow[t]{2}{*}{ Variable } & \multicolumn{2}{|l|}{ Cluster group } & \multirow[t]{2}{*}{ F; P-value } \\
\hline & Group I $(n=18)$ & Group $2(n=3 I)$ & \\
\hline \multirow[t]{3}{*}{ Age (years) Y, O, VO (\%) } & $O=69.6(57.2)$ & $Y=25(45)$ & I \\
\hline & $\mathrm{VO}=79.9(42.8)$ & $O=69.4(32)$ & \\
\hline & & $\mathrm{VO}=79.4(23)$ & \\
\hline Female (\%) & $\mathrm{n}=\mathrm{I} 4(77.7)$ & $n=24(77.4)$ & \\
\hline \multicolumn{4}{|l|}{ Cognitive test } \\
\hline MMSE & $27.9 \pm 1.4$ & $28.4 \pm 0.9$ & I.9; 0.1 \\
\hline FAB & $15.5 \pm 1.5$ & $17.3 \pm 0.8$ & $3.69 ;<0.001$ \\
\hline WAIS (digit span forward and backward) & $97.8 \pm 1.35$ & $99.4 \pm 10.6$ & $1.35 ; 0.5$ \\
\hline Stroop I & $84.3 \pm 11.9$ & $99.9 \pm 15.8$ & $\mathrm{I} .75 ; 0.2$ \\
\hline Stroop II & $117.5 \pm 10.5$ & $107.4 \pm 22.6$ & $4.64 ;<0.0001$ \\
\hline Stroop III & $52.6 \pm 6.4$ & $57 \pm 6.4$ & I.0I; I \\
\hline Stroop interference & $3.73 \pm 7.4$ & $0.34 \pm 7.8$ & I. $12 ; 0.8$ \\
\hline TMT A & $42.3 \pm 11.1$ & $27.2 \pm 11.3$ & I.04; <0.000। \\
\hline TMT B & $100.16 \pm 35.1$ & $53.4 \pm 22.9$ & $2.34 ;<0.0001$ \\
\hline Delta TMT (s) & $57.8 \pm 33.9$ & $26.1 \pm 15.3$ & $4.91 ;<0.0001$ \\
\hline Dubois five word-test & $17.7 \pm 3.1$ & $19.1 \pm 0.9$ & II.2I; 0.02 \\
\hline Clock-drawing test & $6.4 \pm 0.7$ & $6.5 \pm 0.7$ & I.07; 0.4 \\
\hline IADL Part I & $7.3 \pm 1.1$ & $7.1 \pm 2.6$ & I.37; 0.2 \\
\hline Mini GDS & $0.7 \pm 0.3$ & $0.4 \pm 0.4$ & I.I5; 0.07 \\
\hline
\end{tabular}

Note: Values are shown as the mean \pm standard deviation.

Abbreviations: Y, young; O, old; VO, very old; MMSE, Mini Mental State Examination; FAB, Frontal Assessment Battery; WAIS, Wechsler Adult Intelligence Scale; TMT, Trail Making Test; IADL, Instrumental Activities of Daily Living; GDS, Geriatric Depression Scale. 
fragility reflected by a reduction in walking velocity and cognitive fragility reflected by an early alteration in executive function. Nevertheless, the WSC demonstrated that low walking velocity in dual-tasking is an element in cognitive fragility (ie, subjects with blMCI). We suggest that borderline subjects need to be monitored because their cognitive profiles suggest that they are potentially more at risk of developing mild cognitive impairment. Early detection makes it possible to prescribe a program of retraining and/or cognitive stimulation for subjects with slight cognitive decline in order to delay the appearance of new symptoms and/or aggravation of existing cognitive impairment as far as possible.

If we were to hypothesize that these blMCI are going to worsen on the cognitive level, it would mean that alteration in motor performance could occur before detection of cognitive impairment. Could analysis of walking in a dual-task environment be a biomarker of early cognitive impairment? It would be advisable to position that in relation to other concepts, such as that of cognitive reserve ${ }^{50}$ and the ability to distinguish healthy aging from pathologic aging. Indeed, one can suppose that these borderline subjects have a cognitive reserve or possess compensatory mechanisms that enable them to pass cognitive paper-pencil tests, thereby masking their true decrease in intellectual performance during singletasking. However, we know that this cognitive reserve is rapidly overwhelmed in a dual-task situation, revealing motor or cognitive fragility. ${ }^{51,52}$ It is difficult to make a judgment on the pre-eminence of cognitive decline over motor decline, although it is probable that cognitive deterioration, even if undiagnosed, occurs before a change in postural and locomotor patterns. Moreover, it remains difficult to distinguish between a decrease in cognitive performance linked to a pathological state and that related to normal aging, inasmuch as the evolution of mild cognitive impairment is not well understood. ${ }^{3}$ Nevertheless, it would appear that our dual-task conditions contributed to the ability to distinguish between these two categories.

When one investigates human navigation and, in particular, the strategies implemented for moving about, one must also consider the role of vision in walking. The study of intraindividual variability made it possible to verify if Stroop test interference is preserved in dual-task conditions. Group 2 subjects (young, healthy elderly, or with aMCI) were more successful in $\mathrm{DT}_{\text {color }}$ than in $\mathrm{DT}_{\text {word }}$ or $\mathrm{DT}_{\mathrm{B} \& \mathrm{~W}}$, meaning that it appeared easier for these subjects to avoid reading the word than the color. These results tend to go against the Stroop color-word test, in which subjects had the most difficulty refraining from reading the word when they were asked to name the color. In order to maintain a regular walking velocity on the WSC, subjects must anticipate visualizing the targets to process the responses upstream without having to stop. On the WSC, each line has several pieces of information and requires several hesitant jerky movements to process them. We can suppose that the subjects did not have enough time during locomotion to use all of the cues necessary for reading the words. This is the reason they appeared to be less successful during the $\mathrm{DT}_{\text {color }}$ test (interference condition in the paper-pencil version) than during the $\mathrm{DT}_{\text {word }}$ or $\mathrm{DT}_{\mathrm{B} \& \mathrm{~W}^{*}}$

In contrast, we noted no significant difference between dual-task conditions for group 1 subjects with dysexecutive syndrome, meaning that no matter what visual stimuli were used, the complexity of the dual-task was identical for all of the conditions. Interactions between the ocular and locomotor systems could explain why dual-task complexity was identical regardless of the visual stimuli used. During the TMT test, poor locomotor performance is correlated with a decrease in visual ability. ${ }^{14}$ Using the Benton scale, Amieva et $\mathrm{al}^{53}$ showed that alteration of visual memory occurs very early in AD. Thus, the more advanced the cognitive decline, the more the reduction in motor performance in dual-tasking is likely to be linked to a decrease in visual ability. This could also account for why there were no differences in WSC dual-task conditions in group 1. One could suppose that as soon as a subject presenting with cognitive impairment is placed in a visuospatial dual-tasking situation (regardless of the complexity of the task), they will show decreased motor performance.

From a neuroanatomical point of view, Al-Yahya et al ${ }^{17}$ expressed an interest in validating dual-task models to improve our knowledge about walking ability in subjects with dementia and the neuronal mechanisms that control them. Gwin et $\mathrm{al}^{54}$ performed an electroencephalogram on a subject walking on the WSC and noted activation of the anterior cingulate cortex during placement of the foot on the carpet, similar to detection of an error in placing the foot and correction of its trajectory. Moreover, performance of the Stroop test requires involvement of both the dorsolateral prefrontal cortex and the anterior cingulate cortex. ${ }^{55}$ During the WSC test, one could imagine that there is a conflict at the level of the anterior cingulate cortex, which simultaneously manages performance of the cognitive task $\mathrm{k}^{55}$ and correct placement of the foot. ${ }^{54}$ This conflict at the level of the anterior cingulate cortex could be increased in subjects with mdMCI because this cerebral zone is often prematurely deteriorated in patients with dementia.

Scherder et al $^{56}$ believe that one can predict a change in walking control and motor performance in subgroups of 
patients with dementia pathology using the concept of "last in-first out", ie, the neuronal circuits that mature late would be the first to deteriorate in neurodegenerative pathology. Scherder et $\mathrm{al}^{56}$ observed early degeneration of the anterior cingulate cortex and dorsolateral prefrontal cortex in subjects with frontotemporal or vascular dementia which triggered difficulties at a motor level in coordinating complex foot movements and planning movements. Therefore, the motor performance seen in subjects with dysexecutive function from group 1 is likely to be linked to early degeneration of the dorsolateral prefrontal cortices as well as the anterior cingulate cortex, and these subjects would be susceptible to progressive dementia pathology, such as frontotemporal and vascular dementia. Cerebral imaging will be used in a future study to confirm or refute this hypothesis.

Our study has some limitations which should be taken into account. It is based on a small sample of 35 older and 14 younger subjects. Further, it did not include a "gold standard" test to confirm that all subjects with so-called mild cognitive impairment were really cognitively impaired, so it is difficult to compare our results with those of other researchers.

There are several opportunities for future research on this topic. Firstly, it would be interesting to perform these tests in a group of $\mathrm{AD}$ patients to determine whether they perform similarly to the patients in our group 2. Moreover, we plan to follow up our population with repeat testing every year to monitor their cognitive status and confirm the relevance of our tests in the early detection of cognitive disorders. Finally, we suspect that integrating this type of dual-tasking with training programs or physical therapy would delay cognitive and motor decline in the elderly.

\section{Conclusion}

Analysis of spatiotemporal parameters during spontaneous walking does not make it possible to qualify mild cognitive impairment. The WSC test is a good method for early detection of predementia cognitive impairment because it detects subjects with fragile executive function using motor parameters. The test is rapid and simple to perform, and the carpet is adjustable and can be set with increasing difficulty.

\section{Disclosure}

The authors report no conflicts of interest in this work.

\section{References}

1. Waite LM, Grayson DA, Piguet O, Creasey H, Bennett HP, Broe GA. Gait slowing as a predictor of incident dementia: 6-year longitudinal data from the Sydney Older Persons Study. J Neurol Sci. 2005;229-230:89-93.
2. Verghese J, Wang C, Lipton RB, Holtzer R, Xue X. Quantitative gait dysfunction and risk of cognitive decline and dementia. $J$ Neurol Neurosurg Psychiatry. 2007;78(9):929-935.

3. Gauthier S, Reisberg B, Zaudig M, et al. Mild cognitive impairment. Lancet. 2006;367(9518):1262-1270.

4. Petersen RC. Mild cognitive impairment as a diagnostic entity. J Intern Med. 2004;256(3):183-194.

5. Winblad B, Palmer K, Kivipelto M, et al. Mild cognitive impairment beyond controversies, towards a consensus: report of the International Working Group on Mild Cognitive Impairment. J Intern Med. 2004;256(3):240-246.

6. Erk S, Spottke A, Meisen A, Wagner M, Walter H, Jessen F. Evidence of neuronal compensation during episodic memory in subjective memory impairment. Arch Gen Psychiatry. 2011;68(8):845-852.

7. Siu KC, Chou LS, Mayr U, Donkelaar P, Woollacott MH. Does inability to allocate attention contribute to balance constraints during gait in older adults? J Gerontol A Biol Sci Med Sci. 2008;63(12):1364-1369.

8. de Bruin ED, Schmidt A. Walking behaviour of healthy elderly: attention should be paid. Behav Brain Funct. 2010;6:59.

9. Watson NL, Rosano C, Boudreau RM, et al. Executive function, memory, and gait speed decline in well-functioning older adults. J Gerontol A Biol Sci Med Sci. 2010;65(10):1093-1100.

10. Plummer-D'Amato P, Altmann LJ, Reilly K. Dual-task effects of spontaneous speech and executive function on gait in aging: exaggerated effects in slow walkers. Gait Posture. 2011;33(2):233-237.

11. Lundin-Olsson L, Nyberg L, Gustafson Y. "Stops walking when talking" as a predictor of falls in elderly people. Lancet. 1997;349(9052):617.

12. Gillain S, Warzee E, Lekeu F, et al. The value of instrumental gait analysis in elderly healthy, MCI or Alzheimer's disease subjects and a comparison with other clinical tests used in single and dual-task conditions. Ann Phys Rehabil Med. 2009;52(6):453-474.

13. Muir SW, Speechley M, Wells J, Borrie M, Gopaul K, Montero-Odasso M. Gait assessment in mild cognitive impairment and Alzheimer's disease: the effect of dual-task challenges across the cognitive spectrum. Gait Posture. 2012;35(1):96-100.

14. Persad CC, Jones JL, Ashton-Miller JA, Alexander NB, Giordani B. Executive function and gait in older adults with cognitive impairment. J Gerontol A Biol Sci Med Sci. 2008;63(12):1350-1355.

15. Theill N, Martin M, Schumacher V, Bridenbaugh SA, Kressig RW. Simultaneously measuring gait and cognitive performance in cognitively healthy and cognitively impaired older adults: the Basel motor-cognition dual-task paradigm. J Am Geriatr Soc. 2011;59(6): $1012-1018$.

16. Montero-Odasso M, Muir SW, Speechley M. Dual-task complexity affects gait in people with mild cognitive impairment: the interplay between gait variability, dual tasking, and risk of falls. Arch Phys Med Rehabil. 2012;93(2):293-299.

17. Al-Yahya E, Dawes H, Smith L, Dennis A, Howells K, Cockburn J. Cognitive motor interference while walking: a systematic review and meta-analysis. Neurosci Biobehav Rev. 2011;35(3):715-728.

18. Miyake A, Friedman NP, Emerson MJ, Witzki AH, Howerter A, WagerTD. The unity and diversity of executive functions and their contributions to complex "Frontal Lobe" tasks: a latent variable analysis. Cogn Psychol. 2000;41(1):49-100.

19. Yogev-Seligmann G, Hausdorff JM, Giladi N. The role of executive function and attention in gait. Mov Disord. 2008;23(3):329-342.

20. Allali G, Assal F, Kressig RW, Dubost V, Herrmann FR, Beauchet O. Impact of impaired executive function on gait stability. Dement Geriatr Cogn Disord. 2008;26(4):364-369.

21. Montero-Odasso M, Casas A, Hansen KT, et al. Quantitative gait analysis under dual-task in older people with mild cognitive impairment: a reliability study. J Neuroeng Rehabil. 2009;6:35.

22. Fournet N, Mosca C, Moreaud O. Deficits in inhibitory processes in normal aging and patients with Alzheimer's disease: a review. Psychol Neuropsychiatr Vieil. 2007;5(4):281-294. French.

23. Amieva H, Phillips LH, Della Sala S, Henry JD. Inhibitory functioning in Alzheimer's disease. Brain. 2004;127(Pt 5):949-964. 
24. Belleville S, Rouleau N, Van der Linden M. Use of the Hayling task to measure inhibition of prepotent responses in normal aging and Alzheimer's disease. Brain Cogn. 2006;62(2):113-119.

25. Belanger S, Belleville $\mathrm{S}$, Gauthier S. Inhibition impairments in Alzheimer's disease, mild cognitive impairment and healthy aging: effect of congruency proportion in a Stroop task. Neuropsychologia. 2010;48(2):581-590.

26. Dault MC, Geurts AC, Mulder TW, Duysens J. Postural control and cognitive task performance in healthy participants while balancing on different support-surface configurations. Gait Posture. 2001; 14(3):248-255.

27. Grabiner MD, Troy KL. Attention demanding tasks during treadmill walking reduce step width variability in young adults. J Neuroeng Rehabil. 2005;2:25

28. Vallee M, McFadyen BJ, Swaine B, Doyon J, Cantin JF, Dumas D. Effects of environmental demands on locomotion after traumatic brain injury. Arch Phys Med Rehabil. 2006;87(6):806-813.

29. Parsons TD, Courtney CG, Arizmendi B, Dawson M. Virtual Reality Stroop Task for neurocognitive assessment. Stud Health Technol Inform. 2011;163:433-439.

30. Perrochon A, Kemoun G, Watelain E, Berthoz A. [The "ecological Stroop test" : an innovative dual-task concept.] Clin Neurophysiol. 2011;41(4):206. French.

31. Folstein MF, Folstein SE, McHugh PR. "Mini-mental state". A practical method for grading the cognitive state of patients for the clinician. J Psychiatr Res. 1975;12(3):189-198.

32. Dubois B, Slachevsky A, Litvan I, Pillon B. The FAB: a Frontal Assessment Battery at bedside. Neurology. 2000;55(11):1621-1626.

33. Yesavage JA, Brink TL, Rose TL, et al. Development and validation of a geriatric depression screening scale: a preliminary report. J Psychiatr Res. 1982;17(1):37-49.

34. Lawton MP, Brody EM. Assessment of older people: self-maintaining and instrumental activities of daily living. Gerontologist. 1969;9(3): 179-186.

35. Wechsler D. Weschsler Adult Intelligence Scale-III. San Antonio, TX: The Psychological Corporation; 1997.

36. Army Individual Test Battery. Manual of Directions and Scoring. Washington, DC: War Department Adjutant General's Office; 1944.

37. Stroop JR. Studies of interference in serial verbal reactions. J Exp Psychol. 1935;18(6):643-662.

38. Dubois B, Touchon J, Portet F, Ousset PJ, Vellas B, Michel B. "The 5 words": a simple and sensitive test for the diagnosis of Alzheimer's disease. Presse Med. 2002;31(36):1696-1699. French.

39. Troyer AK, Leach L, Strauss E. Aging and response inhibition: Normative data for the Victoria Stroop Test. Neuropsychol Dev Cogn B Aging Neuropsychol Cogn. 2006;13(1):20-35.

40. Lord SR, Clark RD, Webster IW. Visual acuity and contrast sensitivity in relation to falls in an elderly population. Age Ageing. 1991; 20(3):175-181.
41. Ward JH. Hierarchical grouping to optimize an objective function. J Am Stat Assoc. 1963;58:236-244.

42. Hartigan JA. Clustering algorithms. In: Probability and Mathematical Statistics. New York, NY: John Wiley \& Sons; 1975.

43. Maquet D, Lekeu F, Warzee E, et al. Gait analysis in elderly adult patients with mild cognitive impairment and patients with mild Alzheimer's disease: simple versus dual task: a preliminary report. Clin Physiol Funct Imaging. 2010;30(1):51-56.

44. Hausdorff JM, Yogev G, Springer S, Simon ES, Giladi N. Walking is more like catching than tapping: gait in the elderly as a complex cognitive task. Exp Brain Res. 2005;164(4):541-548.

45. Hollands MA, Marple-Horvat DE. Visually guided stepping under conditions of step cycle-related denial of visual information. Exp Brain Res. 1996;109(2):343-356.

46. Chapman GJ, Hollands MA. Evidence for a link between changes to gaze behaviour and risk of falling in older adults during adaptive locomotion. Gait Posture. 2006;24(3):288-294.

47. Chapman GJ, Hollands MA. Evidence that older adult fallers prioritise the planning of future stepping actions over the accurate execution of ongoing steps during complex locomotor tasks. Gait Posture. 2007;26(1):59-67.

48. Di Fabio RP, Zampieri C, Henke J, Olson K, Rickheim D, Russell M. Influence of elderly executive cognitive function on attention in the lower visual field during step initiation. Gerontology. 2005;51(2):94-107.

49. Hommet C, Mondon K, B. B, T. C. [Is alteration of executive functions a factor for frailty in the elderly subject?] Ann Gerontol. 2010;3(3): 175-180.

50. Stern Y. Cognitive reserve. Neuropsychologia. 2009;47(10): 2015-2028.

51. Bherer L, Kramer AF, Peterson MS, Colcombe S, Erickson K, Becic E. Testing the limits of cognitive plasticity in older adults: application to attentional control. Acta Psychol (Amst). 2006;123(3):261-278.

52. Verghese J, Mahoney J, Ambrose AF, Wang C, Holtzer R. Effect of cognitive remediation on gait in sedentary seniors. J Gerontol A Biol Sci Med Sci. 2010;65(12):1338-1343.

53. Amieva H, Le Goff M, Millet X, et al. Prodromal Alzheimer's disease: successive emergence of the clinical symptoms. Ann Neurol. 2008; 64(5):492-498

54. Gwin JT, Gramann K, Makeig S, Ferris DP. Electrocortical activity is coupled to gait cycle phase during treadmill walking. Neuroimage. 2011;54(2):1289-1296.

55. Carter CS, van Veen V. Anterior cingulate cortex and conflict detection: an update of theory and data. Cogn Affect Behav Neurosci. 2007; 7(4):367-379.

56. Scherder E, Eggermont L, Visscher C, Scheltens P, Swaab D. Understanding higher level gait disturbances in mild dementia in order to improve rehabilitation: 'last in-first out'. Neurosci Biobehav Rev. 2011;35(3):699-714.
Clinical Interventions in Aging

\section{Publish your work in this journal}

Clinical Interventions in Aging is an international, peer-reviewed journal focusing on evidence-based reports on the value or lack thereof of treatments intended to prevent or delay the onset of maladaptive correlates of aging in human beings. This journal is indexed on PubMed Central, MedLine, the American Chemical Society's 'Chemical Abstracts Ser-

\section{Dovepress}

vice' (CAS), Scopus and the Elsevier Bibliographic databases. The manuscript management system is completely online and includes a very quick and fair peer-review system, which is all easy to use. Visit http://www.dovepress.com/testimonials.php to read real quotes from published authors. 